\title{
Coexistencia de Pseudomonas aeruginosa y Candida albicans en infecciones nosocomiales en Cartagena de Indias (Colombia)
}

\author{
Javier A. Baena Del Valle, MD. ', Claudio J. Gómez Alegría. PhD. ${ }^{2}$, \\ Doris E. Gómez Camargo. MSc. PhD. ${ }^{3}$
}

1. Médico, Residente de Patología. Joven Investigador e Innovador - COLCIENCIAS, Universidad de Cartagena. Grupo de Investigación UNIMOL.

2. Facultad de Ciencias, Departamento de Farmacia. Universidad Nacional de Colombia. Grupo de Investigación UNIMOL, Bogotá DC, Colombia.

3. Facultad de Medicina, Doctorado en Medicina Tropical. Universidad de Cartagena.

Grupo de Investigación UNIMOL, Cartagena, Colombia.

Correspondencia: degomez@hotmail.com

Recibido: 14/09/2011 - Aceptado: 29/09/2011

\begin{abstract}
Resumen
Las infecciones nosocomiales constituyen un importante problema de salud, cuyos factores de riesgo son hospitalizaciones prolongadas, procedimientos invasivos y tratamientos antimicrobianos de amplio espectro. Pseudomonas aeruginosa y Candida albicans son microorganismos frecuentemente aislados del tracto respiratorio de pacientes gravemente enfermos. Se ha demostrado que estos patógenos pueden tener una interacción de gran significancia en donde las características morfológicas y de virulencia de cada microorganismo se modulan mutuamente aumentando significativamente el riesgo y la severidad de las infecciones urinarias y respiratorias produciendo una alta morbimortalidad. El objetivo de este trabajo fue ilustrar las características microbiológicas y clínicas que son resultado de la presencia conjunta de P. aeruginosa y C. albicans en pacientes gravemente enfermos en hospitales de Cartagena de Indias (Colombia). En todos los casos se destaca un proceso bacteriano inicial, en este caso causado por $P$. aeruginosa, que fue tratado de acuerdo a la susceptibilidad antimicrobiana encontrada y al disminuir el agente bacteriano responsable se dio lugar al crecimiento de C. albicans y al desarrollo de una nueva infección que empeoró la condición clínica de estos pacientes.

Las infecciones conjuntas entre $P$. aeruginosa y $C$. albicans siempre se deben sospechar en un paciente hospitalizado, especialmente en unidades de cuidados intensivos y cuando hagan uso de sondas, catéteres y otros materiales para estudios invasivos, pues estos microorganismos son de naturaleza oportunista y claramente pueden empeorar el pronóstico y llevar a complicaciones a pacientes que han sido hospitalizado por causas diferentes o enfermedades de baja complejidad, prolongando el tiempo de hospitalización y aumentando costos.

Palabras clave: Infección hospitalaria, Pseudomonas aeruginosa, Candida albicans, técnica del ADN polimorfo amplificado aleatorio, pruebas antimicrobianas de difusión por disco.
\end{abstract}




\section{Abstract \\ Coexistence of Pseudomonas Aeruginosa and Candida albicans in hospital-acquired infections in Cartagena (Colombia)}

Nosocomial infections are a major health problem, whose risk factors include prolonged hospitalization, invasive procedures and broad-spectrum antimicrobial treatments. Pseudomonas aeruginosa and Candida albicans are commonly isolated microorganisms from the respiratory tract of seriously ill patients. It has been shown that these pathogens may have an interaction of great significance where the morphological characteristics and virulence of each organism is significantly expanding mutually modulate risk and severity of urinary and respiratory infections, causing high morbidity and mortality. The aim of this paper is to illustrate the clinical and microbiological characteristics that result from the presence of both $P$. aeruginosa and $C$. albicans in critically ill patients in hospitals in Cartagena de Indias (Colombia). In all cases, initial bacterial process (in this case caused by $P$. aeruginosa) was treated according to antimicrobial susceptibility and found to decrease the bacterial agent, led to the growth of $C$. albicans and the development of a new infection that worsened the clinical condition of these patients. Joint infections $P$. aeruginosa and $C$. albicans should always be suspected in a hospitalized patient, especially in intensive care units where they use the tubes, catheters and other invasive study materials, as these organisms are opportunistic in nature and clearly can worsen the prognosis and lead to complications in patients who have been hospitalized for various causes or diseases of low complexity, a longer hospital stay and increasing costs.

Keywords: Hospital Infections, Pseudomonas aeruginosa, Candida albicans, random amplified polymorphic DNA technique, disk diffusion antimicrobial tests.

\section{Introducción}

Las infecciones nosocomiales constituyen un importante problema de salud, cuyos factores de riesgo son hospitalizaciones prolongadas, procedimientos invasivos y tratamientos antimicrobianos de amplio espectro (1). A pesar de los esfuerzos para prevenir estas complicaciones no se ha logrado disminuir notablemente su ocurrencia en pacientes críticamente enfermos generando altos gastos económicos y aumento en la mortalidad (2 - 3).

Pseudomonas aeruginosa y Candida albicans son microrganismos frecuentemente aislados del tracto respiratorio de pacientes gravemente enfermos con necesidad de ventilación mecánica y de pacientes con infecciones de vías urinarias, especialmente con cateterización uretral $(4-5)$.

$P$. aeruginosa es responsable de graves infecciones respiratorias y de vías urinarias (6-7). Siendo el segundo patógeno más frecuentemente aislado de pacientes con neumonía asociada a ventilador (8) y causante de una infección crónica de difícil erradicación en pacientes con fibrosis quística (FQ) (9). De la misma forma, es responsable de infecciones del tracto urinario difíciles de tratar debido a la rápida capacidad para adquirir resistencia a los antimicrobianos (10). Por otro lado, la presencia de $C$. albicans en especímenes respiratorios es considerada una colonización en el paciente inmunocompetente, pero este hongo está presente en el $17 \%$ al $28 \%$ de pacientes hospitalizados en unidades de cuidados intensivos (UCI) que reciben ventilación mecánica y se asocia a una peor evolución clínica, aumentando dramáticamente la mortalidad hospitalaria (11).

Se ha demostrado que $P$. aeruginosa y $C$. albicans pueden tener una interacción patogénica de gran significancia en donde las características morfológicas y de virulencia de cada microorganismo se modulan mutuamente aumentando significativamente el riesgo y la severidad de las infecciones urinarias y respiratorias, produciendo una alta morbimortalidad (12 - 14). P. aeruginosa es capaz de producir una molécula de señalización que puede inhibir las formas filamentosas de $C$. albicans, además se organiza en forma de biopelícula alrededor de los filamentos 
de $C$. albicans erradicando al hongo. Sin embargo, $P$. aeruginosa puede unirse a la forma levaduriforme de C. albicans, pero sin poder eliminarla $(15-16)$.

Los cuatro reportes de casos presentados en este escrito ilustran las características microbiológicas y clínicas que son resultado de la presencia conjunta de P. aeruginosa y $C$. albicans en pacientes gravemente enfermos en hospitales de Cartagena de Indias (Colombia).

\section{Materiales y métodos}

Se estudiaron pacientes hospitalizados en UCI de hospitales de la ciudad de Cartagena de Indias cuyas muestras y reportes clínicos fueron remitidos a nuestro laboratorio para análisis microbiológico y en los que se sospecho de coexistencia de Pseudomonas aeruginosa y Candida albicans en infecciones nosocomiales.

\section{Resultados}

Caso 1. Un adolescente masculino de 16 años de edad con diagnóstico de FQ desde su niñez que fue admitido al servicio de urgencias por presentar disnea y fiebre de una semana de evolución. Se encontró en malas condiciones generales, con crepitantes en ambas bases pulmonares y franca dificultad respiratoria. Los paraclínicos reportaron leucocitosis con neutrofilia, ordenando inmediatamente hospitalización y tratamiento con meropenem $(30 \mathrm{mg} / \mathrm{Kg}$ cada 8 horas) y cefepime ( $1 \mathrm{gr}$ cada 12 horas), pues se supo por cultivos realizados anteriormente que el paciente estaba infectado de forma crónica por $P$. aeruginosa. En el curso de la hospitalización, se tomo una muestra de esputo que fue cultivada en el laboratorio clínico del hospital, encontrando abundante crecimiento de $P$. aeruginosa sensible a cefepime, ciprofloxacina, amikacina, gentamicina, meropenem y resistente a eritromicina, cefotaxime, ceftriaxone y piperacilina/ tazobactam. Durante el tratamiento antimicrobiano, la fiebre y los síntomas iniciales no disminuyeron, por lo tanto se envió una muestra de esputo al Laboratorio de Investigaciones de la Facultad de Medicina de la Universidad de Cartagena, cuyo cultivo reveló crecimiento de $P$. aeruginosa y abundantes levaduras, Figura 1, a y b. La identificación de $C$. albicans se basó en la observación al microscopio, tests fenotípicos y en prueba del tubo germinal, Figura 2. Paralelamente se realizó un análisis molecular (RAPD - PCR) para comparar el aislado bacteriano con una cepa de referencia de $P$. aeruginosa., Figura 1, c. Dos días más tarde se recibió una segunda muestra, analizada de igual forma, en la que se obtuvo crecimiento de P. aeruginosa. y formas filamentosas de C. albicans. Inmediatamente se añadió Voriconazol
A.

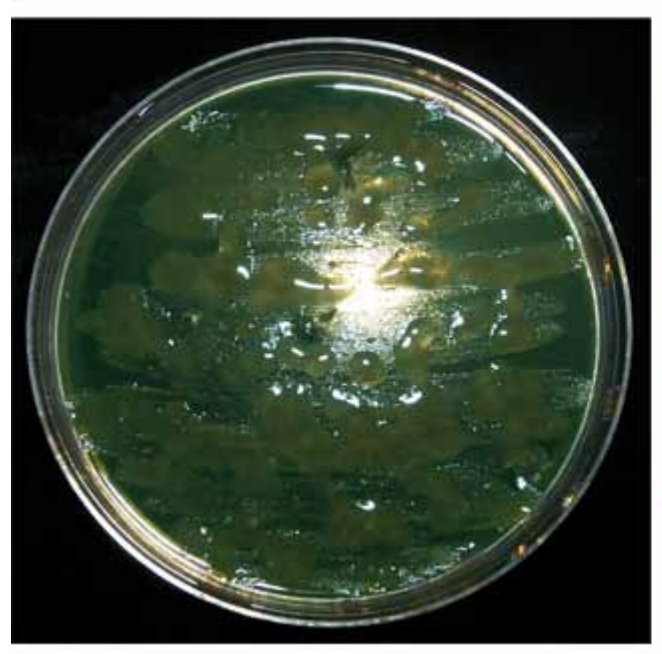

B.

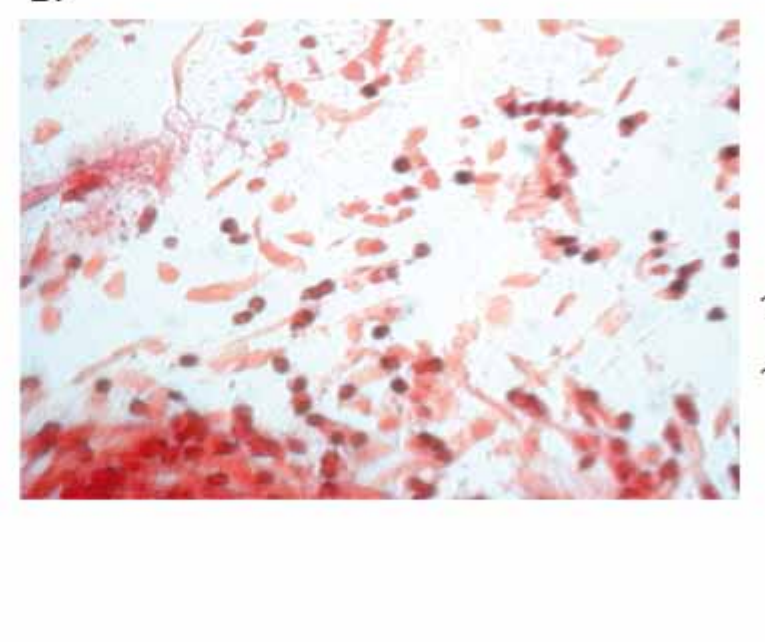

C. M 12

500 1000 500

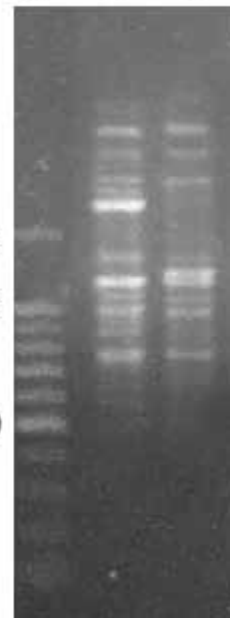

Figura 1. Caso 1. Cultivo en placa de agar LB con abundante crecimiento de $P$. aeruginosa con fenotipo mucoide (A). Extendido que revela $P$. aeruginosa y levaduras de $C$. albicans (Tinción de Gram, 100X) (B). Caracterización molecular por RAPD - PCR de la cepa de P. aeruginosa del paciente (1) y una cepa de referencia (2). Marcador de peso molecular (M) expresado en pares de bases. 


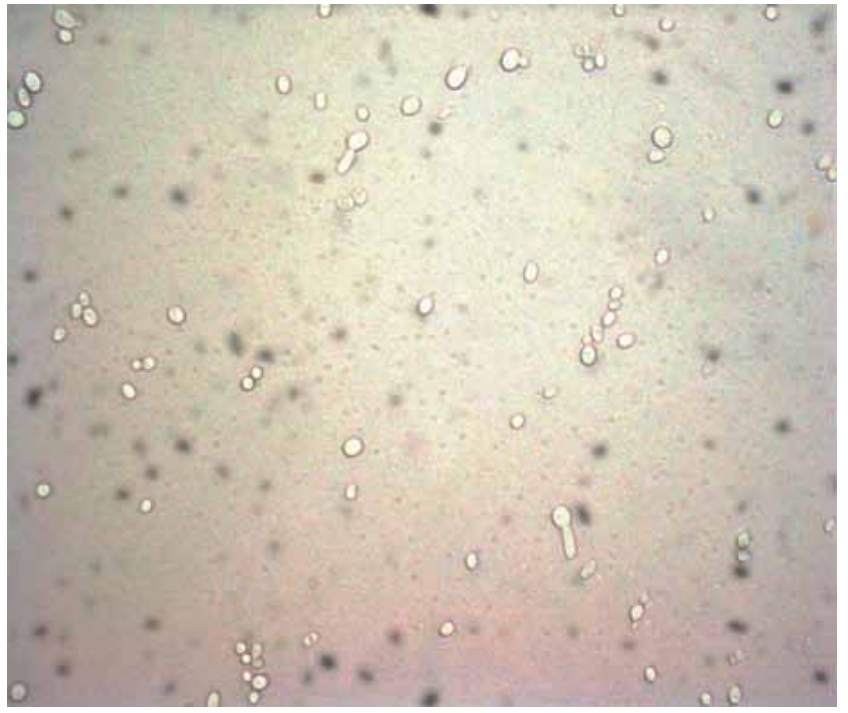

Figura 2. Prueba del tubo germinal de uno de los aislados de C. albicans. $100 X$.

al esquema antimicrobiano, desapareciendo la fiebre y disminuyendo la disnea y fatiga del paciente. Semanas más tarde es llevado nuevamente al servicio de urgencias por presentar dificultad respiratoria aguda y fallece de hemorragia pulmonar algunas horas después.

Caso 2. Una paciente femenina de 19 años de edad quien consultó al servicio de urgencias por disfagia, malestar general y tos con expectoración hialina de cinco días de evolución. Al examen físico se evidenció hipertrofia de amígdalas con abundante exudado purulento e hiperémicas. Se registraron signos vitales dentro de parámetros normales. Siguiendo un diagnostico inicial de faringoamigdalitis bacteriana se instaura tratamiento con piperacilina/ tazobactam (12/1,5gr cada 8 horas). Dos días después, la paciente presentó disnea y se auscultaron crépitos basales bilaterales. Una radiografía de tórax reportó infiltrados algodonosos bilaterales difusos y borramiento de ángulos costofrénicos, una tomografía axial computarizada (TAC) de tórax reveló broncograma aéreo y compromiso parenquimatoso pulmonar bilateral + derrame pleural. Los exámenes de laboratorio evidenciaron leucocitos en 6.800/ $\mathrm{mm}^{3}$, Proteína $\mathrm{C}$ reactiva cualitativa positiva y una saturación periférica de oxigeno $\left(\mathrm{SpO}_{2}\right)$ de $97 \%$. Por lo anterior se trasladó a UCI con diagnostico de neumonía complicada con derrame pleural, sospechando presencia de Staphilococcus aureus como agente etiológico. El estado general de la paciente se empeoró presentando taquicardia, taquipnea y trombocitopenia. A pesar de presentar hemocultivos y cultivos de exudado faríngeo negativos, se cambió el tratamiento antimicrobiano a meropenem ( $1 \mathrm{gr}$ cada 8 horas) y linezolid ( $10 \mathrm{mg} / \mathrm{kg}$ cada 8 horas). Un día después, un doppler de cuello reveló trombosis subaguda de dos tercios superiores de venas yugulares internas bilaterales y gas en región cervical, se hace diagnóstico de síndrome de Lemierre y disfunción orgánica múltiple por sepsis faringoamigdalar y respiratoria. Debido al empeoramiento del compromiso de tórax se realizó intubación y se añadió clindamicina al tratamiento.

Muestras provenientes de faringe y aspirado del tubo endotraqueal fueron enviadas a nuestro laboratorio en donde se cultivaron en placas de agar y caldo LB (Luria Bertani Sigma-Aldrich, Allenton PA, $\mathrm{EU})$, posteriormente las colonias fueron analizadas por tinción de gram en donde se observaron abundantes levaduras gemantes y escasos bacilos gram negativos, Figura 3 A. La presencia de P. aeruginosa. se comprobó utilizando medios de cultivo selectivos y RAPD - PCR, Figura 3, C. Se realizó test de tubo germinal para estudiar la naturaleza de $C$. albicans. Posteriormente se realizaron subcultivos para separar los microorganismos con el fin de efectuar pruebas de susceptibilidad antimicrobiana. Se elaboró un sensidisco conteniendo $25 \mu \mathrm{g}$ de Fluconazol y se desarrolló un test de susceptibilidad antifúngica para levaduras según Barry y Cols (17) que reveló sensibilidad a este fármaco Figura 3, B.

Inmediatamente se instauró tratamiento con fluconazol ( $400 \mathrm{mg}$ cada 12 horas), observando dos días después mejoría del infiltrado pulmonar y leucocitos dentro de parámetros normales. Al concluir el tratamiento antifúngico se continuó con meropenem en monoterapia y al día siguiente se realizó extubación por presentar mejoría, retirando adicionalmente sonda vesical y catéter central. La paciente fue trasladada a sala general sin signos de respuesta inflamatoria sistémica y se inició tratamiento con moxifloxacina vía oral ( $400 \mathrm{mg}$ cada 24 horas), dos días después de concluir tratamiento la paciente fue dada de alta con orden para consultas y estudios de control domiciliarios. 
A.

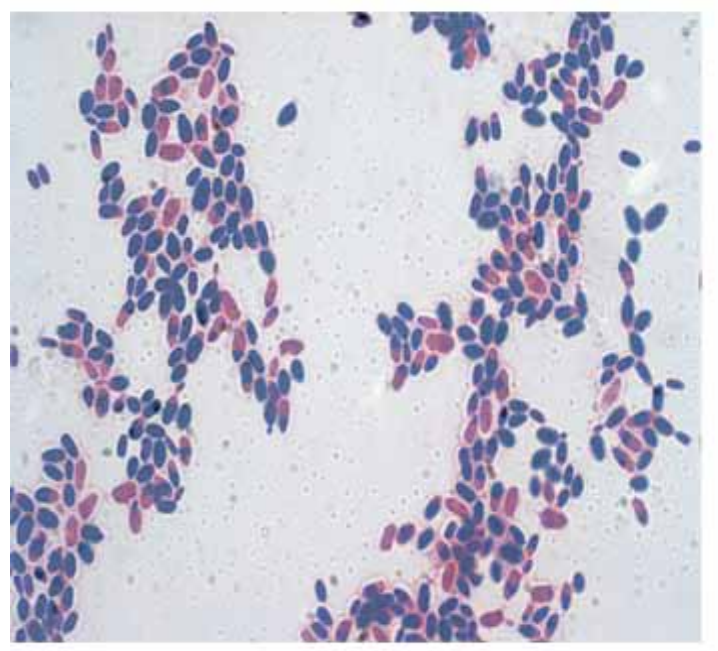

B.

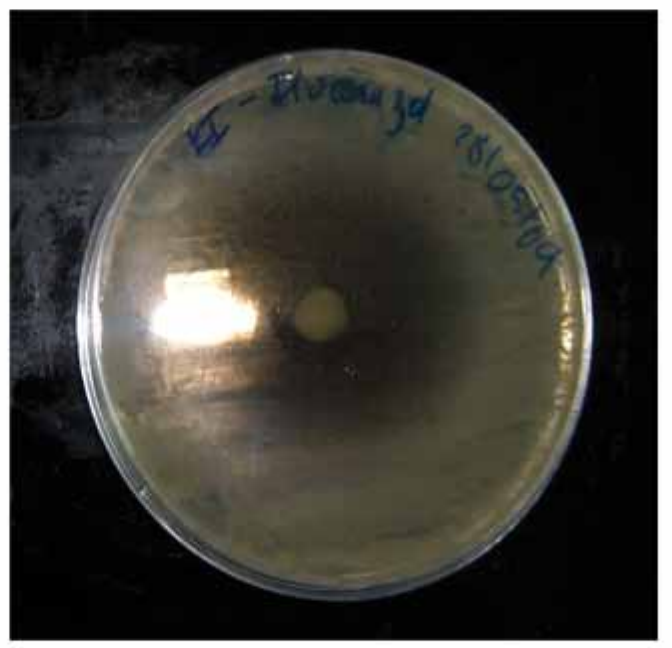

C.

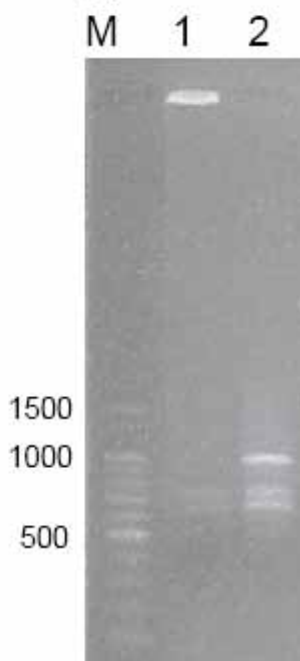

Figura 3. Caso 2. Extendido que revela levaduras de C. albicans y escasos bacilos gram negativos (P. aeruginosa) (Tinción de Gram, 100X) (A). Prueba de Susceptibilidad antifúngica de difusión por disco de Fluconazol (25 $\mu \mathrm{g})$ (B). Caracterización molecular por RAPD - PCR de la cepa de $P$. aeruginosa del paciente (1) y una cepa de referencia (2). Marcador de peso molecular (M) expresado en pares de bases.

Caso 3. Un paciente masculino de 67 años de edad con hospitalización domiciliaria, hipertenso son secuelas de isquemia cerebral, vejiga neurogénica, síndrome convulsivo e infecciones de vías urinarias recurrente por Pseudomonas aeruginosa documentada por urocultivos previos. Ingresó al servicio de urgencias por fiebre, orina fétida, oliguria, edema facial matutino, tos con abundante expectoración y distensión abdominal. Al examen físico se evidenció facies de enfermedad crónica, sonda vesical a cistoflo drenando orina colúrica con abundante sedimento blanquecino y secreción purulenta con eritema en estoma de gatrostomía. Se hizo impresión diagnóstica de infección de vías urinarias y se inició tratamiento con meropenem (1gr I.V. cada 8 horas). Un uroanálisis reveló bacterias ++, 37 leucocitos por campo y 4 hematíes por campo. El hemograma reportó leucocitos en $6.200 / \mathrm{mm}^{3}$, hemoglobina en $10,2 \mathrm{gr} / \mathrm{dl}$, hematocrito $31,2 \%$. Los resultados preliminares de un urocultivo realizado durante su hospitalización reportó crecimiento mayor a 100.000 UFC de cocos gram negativos. Dos días después del ingreso y en espera de resultados de hemocultivos, el paciente presentó tendencia hacia la hipotensión de probable etiología séptica, por lo que se cataloga como sepsis severa por foco urinario. Se administró bolo I.V. de cristaloides al cual respondió favorablemente, continuando igual tratamiento sin necesidad de soporte vasopresor.
Los familiares del paciente enviaron una muestra de orina a nuestro laboratorio para realización de uroanálisis y urocultivo. Se encontró una orina turbia, al análisis microscópico se observaron campos llenos de leucocitos, hematíes 25 -36 por campo, células epiteliales + , bacterias ++ , levaduras ++ , levaduras en gemación ++ , hifas +++ , micelios ++++ , moco + . El urocultivo reportó crecimiento mayor a 100.000 UFC de $P$. aeruginosa. sensible a gentamicina, amikacina, ciprofloxacino, levofloxacino, meropenem, imipenem y resistente a penicilina, eritromicina, ceftriaxone, cefalexina, cefepime, nitrofurantoína (Figura 4, B). También se observó crecimiento de levaduras y formas filamentosas de C. albicans, confirmando su naturaleza por análisis microscópico, fenotípico y test de tubo germinal, Figura 4, A.

El paciente completó tratamiento con meropenem y se ańadió fluconazol ( $400 \mathrm{mg}$ cada 12 horas), mejorando de forma significativa su condición clínica, se dio de alta continuando con hospitalización domiciliaria. Días después, un cultivo de punta de sonda de gastrostomía mostró crecimiento abundante de $P$. aeruginosa. con patrón de susceptibilidad antimicrobiana idéntico al encontrado en nuestro laboratorio.

Caso 4. Un paciente masculino de 80 años de edad, quien ingresó al servicio de urgencias por hemorragia subaracnoidea y hematoma subdural agudo. La imagenología inicial reveló hematoma subdural frontoparietotemporal izquierdo sin 
A.

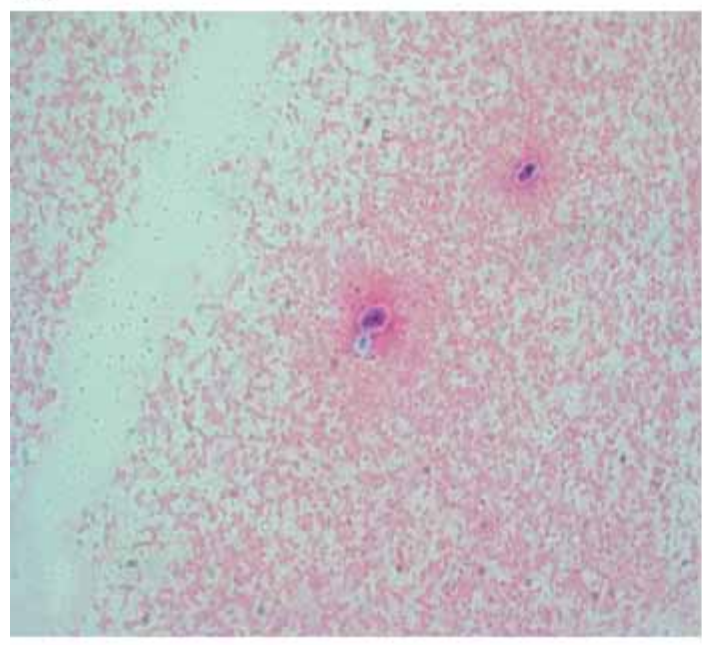

B.

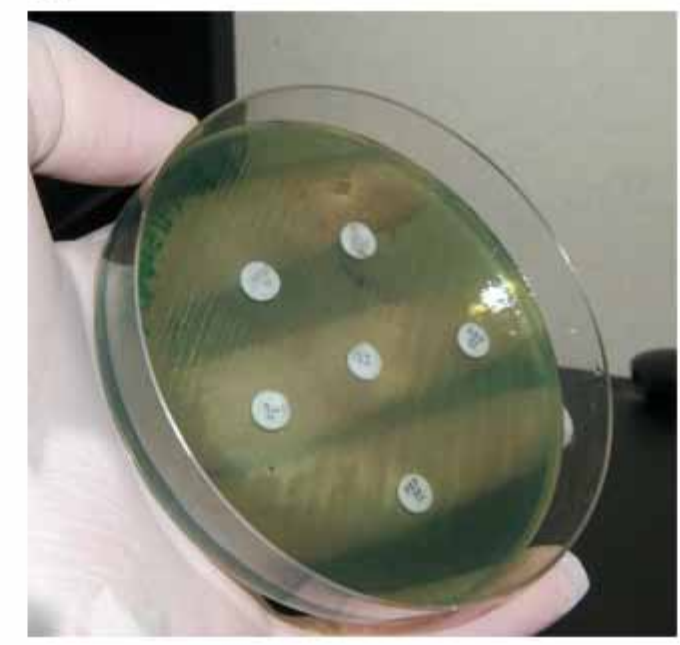

C.

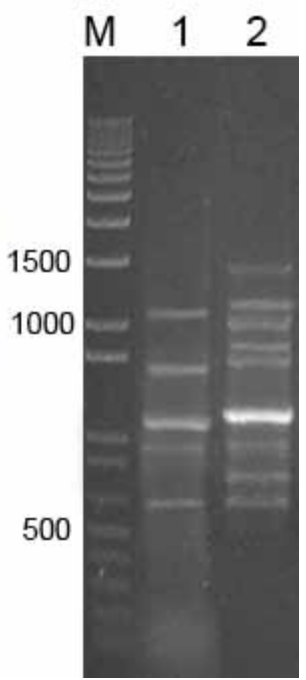

Figura 4. Caso 3. Extendido que revela levaduras gemantes de C. albicans rodeadas por abundante cantidad de P. aeruginosa (Tinción de Gram, 100X) (A). Prueba de Susceptibilidad antimicrobiana de difusión por disco sobre el aislado de P. aeruginosa (B). Caracterización molecular por RAPD - PCR de la cepa de P. aeruginosa del paciente (1) y una cepa de referencia (2). Marcador de peso molecular (M) expresado en pares de bases.

cambios significativos y ateromatosis calcificada vertebrobasilar y carotídea. Debido al deterioro de la condición clínica del paciente a una semana del ingreso, se le realizó un hemocultivo que reportó $P$. aeruginosa sensible a amikacina, aztreonam, cefepime, cefoperazona/sulbactam, ciprofloxacina, gentamicina, imipenem, meropenem, piperacilina/tazobactam y doripenem. Inmediatamente se inicio tratamiento con ertapenem ( 1 gr cada 24 horas). A los 15 días de este suceso, por deterioro de parámetros respiratorios se decidió tomar una muestra de aspirado traqueal para cultivo en el cual se encontró crecimiento de Pseudomonas aeruginosa con igual patrón de susceptibilidad antimicrobiana a la encontrada en sangre. Ese mismo día, una muestra de orina fue analizada y cultivada encontrando crecimiento de la misma bacteria.

La condición del paciente no mejoró durante el tratamiento antimicrobiano empleado, por eso un muestra de orina fue enviada a nuestro laboratorio para ser analizada. Se observaron leucocitos 16 - 20 por campo, hematíes 25 - 30 por campo, células epiteliales + , bacterias ++ , uratos amorfos + , levaduras en gemación ++. El urocultivo reveló abundante crecimiento de levaduras gemantes y formas filamentosas de $C$. albicans y escasos bacilos gram negativos, cuyo análisis por biología molecular (RAPD - PCR) y métodos bacteriológicos convencionales confirmaron P. aeruginosa., Figura 5, A y C.
El informe inmediato de estos resultados hizo que los facultativos tomaran la decisión de iniciar terapia con fluconazol ( $400 \mathrm{mg}$ cada 12 horas), mientras en el laboratorio de investigaciones de la Facultad de Medicina de la Universidad de Cartagena se realizó un fungigrama con un sensidisco conteniendo 25 $\mu \mathrm{g}$ de Fluconazol, como se detalló anteriormente, el cual reportó resistencia a este fármaco, Figura $5 \mathrm{~B}$, dicho hallazgo era compatible con la falta de respuesta del paciente al tratamiento después de añadir el antifúngico; por esta razón se realizó otra prueba de susceptibilidad empleando anfotericina B e itraconazol con los cuales se encontró sensibilidad Figura 6, aunque el paciente falleció algunas horas después de este hallazgo por falla orgánica multisistémica debido a una sepsis severa.

\section{Discusión}

C. albicans es un hongo que hace parte de la flora microbiana comensal humana, pero también puede actuar como un patógeno oportunista, causando graves infecciones que pueden llegar a ser mortales $(13,14)$. La patogenicidad de este microorganismo está determinada en gran parte por la habilidad para cambiar su morfología entre levaduras, hifas y pseudohifas $(17-21)$.

En diversos sitios del cuerpo humano $C$. albicans coexiste con otros microorganismos y estos pueden 
A.

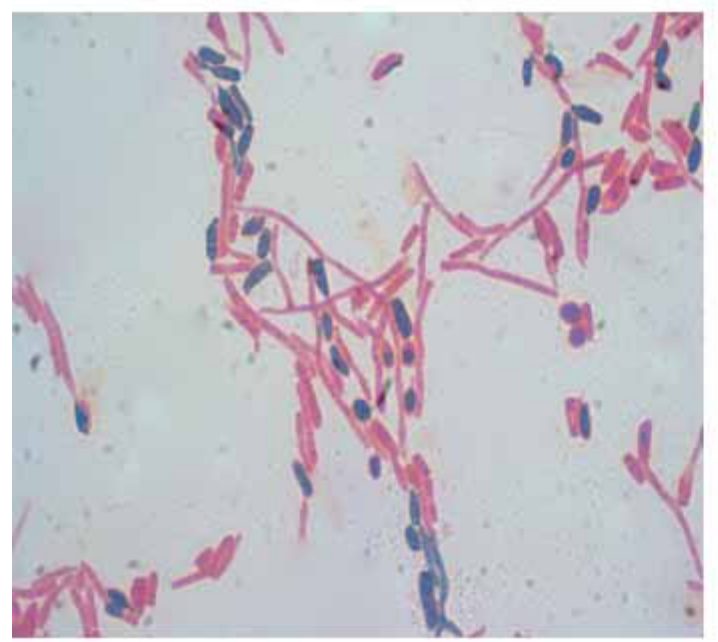

B.

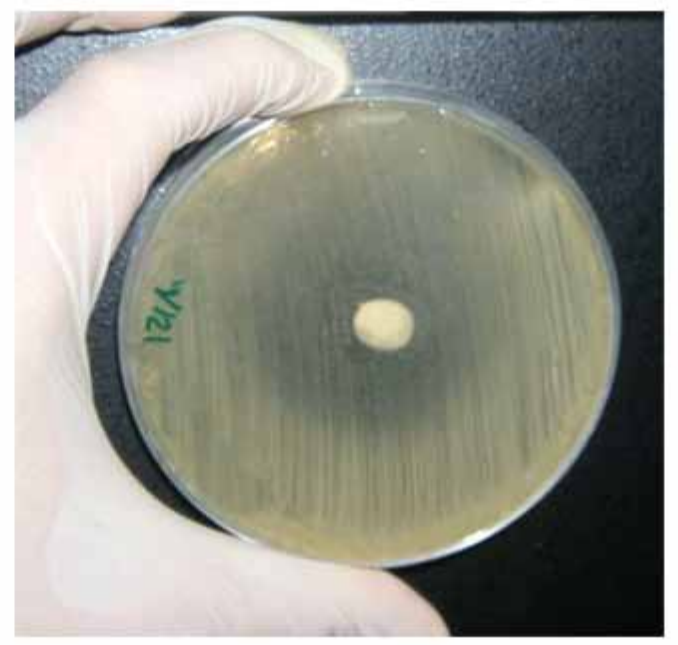

C.

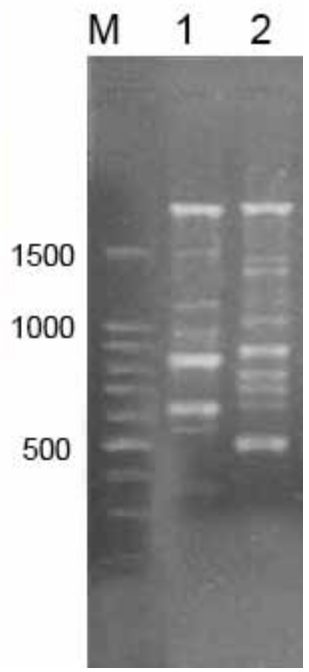

Figura 5. Caso 4. Extendido que revela levaduras y formas filamentosas de $C$. albicans y escasos bacilos gram negativos (P. aeruginosa) (Tinción de Gram, 100X) (A). Prueba de Susceptibilidad antifúngica de difusión por disco de Fluconazol (25 $\mu \mathrm{g})$ reportando resistencia (B). Caracterización molecular por RAPD - PCR de la cepa de P. aeruginosa del paciente (1) y una cepa de referencia (2). Marcador de peso molecular (M) expresado en pares de bases.

A.

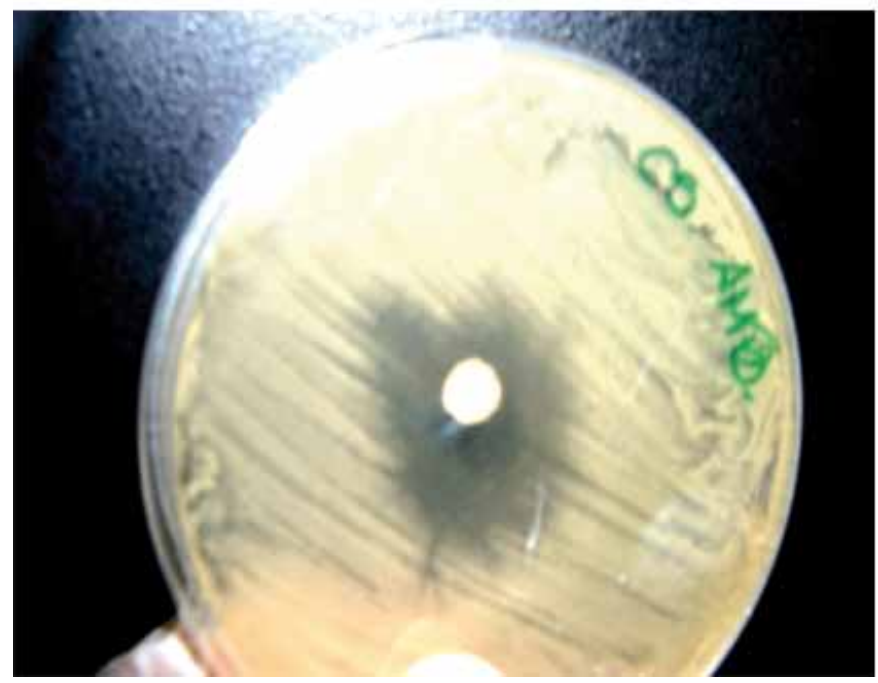

B.

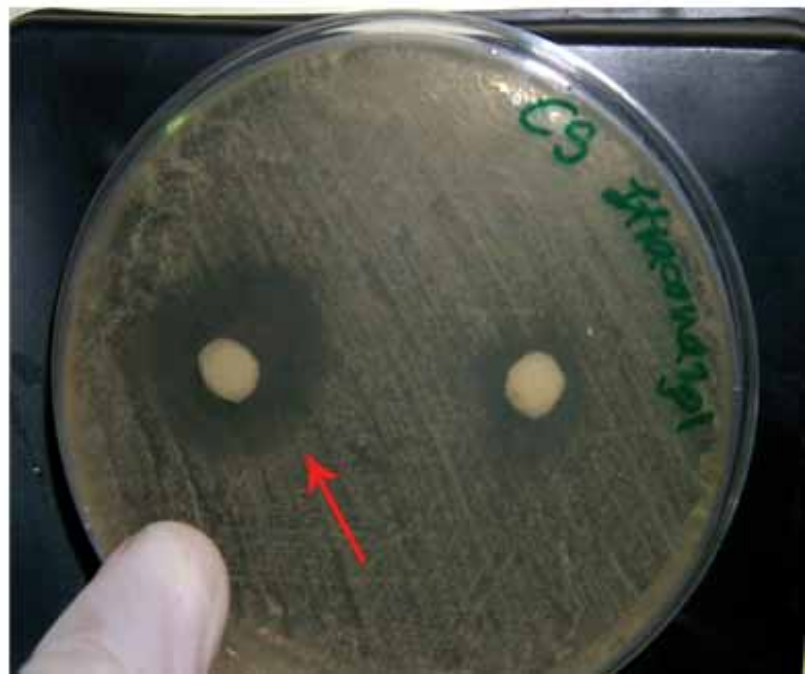

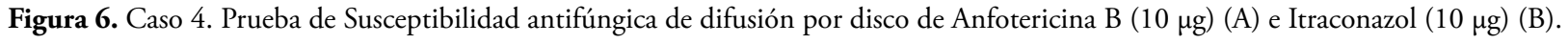

controlar el crecimiento de este hongo $(22,23)$. C. albicans y $P$. aeruginosa son frecuentemente coaislados del esputo de pacientes con $\mathrm{FQ}$, secreciones respiratorias de pacientes intubados y en infecciones del tracto urinario $(7,10,12,24)$.

Los casos aquí descritos son ejemplos de infección conjunta por $P$. aeruginosa y C. albicans, en los cuales se destaca un proceso bacteriano inicial (en este caso causado por $P$. aeruginosa) que fue tratado de acuerdo a la susceptibilidad antimicrobiana encontrada y al disminuir el agente bacteriano responsable, dio lugar al crecimiento de C. albicans y al desarrollo de una nueva infección que empeoró la condición clínica de estos pacientes. En algunos estudios se ha reportado que en el escenario de un proceso infeccioso, el crecimiento de C. albicans se observa después que las bacterias han sido erradicadas y algunos ensayos demostraron que la proliferación del hongo era inhibida in vitro por la cepa de $P$. aeruginosa coaislada $(12,25)$.

$P$. aeruginosa es un patógeno que posee una gran variedad de factores de virulencia y determinantes 
que condicionan su relación con el huésped y con otros microorganismos, causando una gran cantidad de infecciones asociadas con quemaduras, catéteres, implantes y estados de inmunosupresión (26 - 28). Inicialmente Kerr y Cols demostraron que $P$. aeruginosa podía inhibir el crecimiento de Candida spp $(12,25)$. Sin embargo, años más tarde Hogan y Kolter observaron que las formas levaduriformes de $C$. albicans eran resistentes a esta bacteria en un estudio utilizando $C$. albicans mutantes tup1 (constitutivamente filamentosa), en donde adicionalmente descubrieron que la formación de una biopelícula y las propiedades del sistema de quórum sensing son necesarias para el antagonismo entre la bacteria y el hongo microscópico, que le permite a $P$. aeruginosa obtener nutrientes de los filamentos de C. albicans y mantener el equilibrio en la patogénesis (24).

Lo anterior está ilustrado en el caso 1, en donde al inicio del cuadro clínico del paciente se logran aislar levaduras de $C$. albicans y días más tarde, en el curso de la terapia antimicrobiana para controlar la infección por $P$. aeruginosa, se encuentran abundantes formas filamentosas del hongo en una nueva muestra de esputo, las cuales pudieron desarrollarse tal vez por la disminución en el conteo de bacterias que ejercían una acción nociva sobre este. En general en los cuatro casos descritos, la presencia de formas filamentosas de $C$. albicans se relaciona con el empeoramiento de la condición del paciente lo cual es consistente con estudios realizados anteriormente en donde los cambios fenotípicos hacia formas filamentosas contribuyen a su adaptación y expresión de genes de virulencia responsables del proceso patológico en el huésped $(13,29,30)$.

Es interesante resaltar los hallazgos en el cultivo del caso 4, en donde la microfotografía permite observar algunas levaduras en gemación rodeadas por $P$. aeruginosa en forma de biopelícula (Figura 6a), esta estructura ha sido observada en microfotografías electrónicas de pacientes con este tipo de infecciones $(24,31)$ y puede representar el interesante fenómeno de erradicación selectiva de $P$. aeruginosa sobre pseudohifas de C. albicans.

La resistencia de $C$. albicans a los azoles, especialmente al fluconazol, es otro tópico que se debe considerar en pacientes gravemente enfermos, como los presentados en este reporte. Debido al amplio uso de este medicamento para tratar candidiasis, la frecuencia de fallas en el tratamiento por resistencia farmacológica ha aumentado significativamente en los últimos ańos $(32-34)$. Es por esta razón que en este tipo de pacientes con factores de riesgo para infección por cepas resistentes, se deben hacer pruebas de susceptibilidad antifúngica para mayor seguridad del tratamiento antimicótico.

Las infecciones conjuntas entre $P$. aeruginosa y $C$. albicans siempre se deberán sospechar en un paciente hospitalizado, especialmente en unidades de cuidados intensivos y cuando hagan uso de sondas, catéteres y otros materiales para estudios invasivos, pues estos microorganismos son de naturaleza oportunista y claramente pueden empeorar el pronóstico y llevar a complicaciones a pacientes que han sido hospitalizado por causas diferentes o enfermedades de baja complejidad, prolongando el tiempo de hospitalización y aumentando costos.

\section{Referencias}

1. Smith RL. Prevention of infection in the intensive care unit. Curr Opin Infect Dis 2006; 19(4):323-6.

2. Fridkin SK, Welbel SF, Weinstein. Magnitude and prevention of nosocomial infections in the intensive care unit. Infect Dis Clin North Am 1997; 11(2):479-96.

3. Division of Healthcare Quality Promotion, National Center for Infectious Diseases, Centers for Disease Control and Prevention, Public Health Service, US Department of Health and Human Services. National Nosocomial Infections Surveillance (NNIS) System Report, data summary from January 1992 through June 2004, issued October 2004. Am J Infect Control 2004; 32:470-485.

4. Craven DE. Preventing ventilator-associated pneumonia in adults: Sowing seeds of change. Chest 2006; 130:251-260.

5. Warren JW. Catheter-associated urinary tract infections. Int J Antimicrob Agents 2001; 17(4):299-303.

6. Niederman MS. Use of broad-spectrum antimicrobials for the treatment of pneumonia in seriously ill patients: maximizing clinical outcomes and minimizing selection of resistant organisms. Clin Infect Dis 2006; 42 Suppl 2:S72-81.

7. Shigemura K, Arakawa S, Sakai Y, Kinoshita S, Tanaka K, Fujisawa M. Complicated urinary tract infection caused by Pseudomonas aeruginosa in a single institution (1999-2003). Int J Urol 2006; 13(5):538-42.

8. Kollef MH, Morrow LE, Niederman MS, Leeper KV, Anzueto A, Benz-Scott L, Rodino FJ. Clinical characteristics and treatment patterns among patients with ventilator-associated pneumonia. Chest 2006; 129(5):1210-8.

9. Lanotte P, Watt S, Mereghetti L, Dartiguelongue N, Rastegar-Lari A, Goudeau A, Quentin R. Genetic features of Pseudomonas aeruginosa isolates from cystic fibrosis patients compared with those of isolates from other origins. J Med Microbiol 2004; 53(Pt 1):73-81. 
10. Horii T, Muramatsu H, Morita M, Maekawa M. Characterization of Pseudomonas aeruginosa isolates from patients with urinary tract infections during antibiotic therapy. Microb Drug Resis 2003; 9(2):223-9.

11. Delisle MS, Williamson DR, Perreault MM, Albert M, Jiang X, Heyland DK. The clinical significance of Candida colonization of respiratory tract secretions in critically ill patients. J Crit Care 2008; 23(1):11-7.

12. Kerr JR. Suppression of fungal growth exhibited by Pseudomonas aeruginosa. J Clin Microbiol 1994; 32(2):525-7.

13. Liu Y, Kang Y, Yokoyama K, Gonoi T, Mikami Y. Molecular differentiation and antifungal susceptibility of Candida albicans isolated from patients with respiratory infections in Guiyang Medical College Hospital, China. Nippon Ishinkin Gakkai Zasshi 2009; 50(3):175-8.

14. Pierce GE. Pseudomonas aeruginosa, Candida albicans, and device-related nosocomial infections: implications, trends, and potential approaches for control. J Ind Microbiol Biotechnol 2005; 32(7):309-18.

15. Hogan DA, Vik A, Kolter R. A Pseudomonas aeruginosa quorum-sensing molecule influences Candida albicans morphology. Mol Microbiol 2004; 54(5):1212-23.

16. Roux D, Gaudry S, Dreyfuss D, El-Benna J, de Prost N, Denamur E, Saumon G, Ricard JD. Candida albicans impairs macrophage function and facilitates Pseudomonas aeruginosa pneumonia in rat. Crit Care Med 2009; 37(3):1062-7.

17. Barry AL, Brown SD. Fluconazole disk diffusion procedure for determining susceptibility of Candida species. J Clin Microbiol 1996; 34(9):2154-7.

18. Brand A, Barnes JD, Mackenzie KS, Odds FC, Gow NA. Cell wall glycans and soluble factors determine the interactions between the hyphae of Candida albicans and Pseudomonas aeruginosa. FEMS Microbiol Lett 2008; 287(1):48-55.

19. Nseir S, Ader F. Pseudomonas aeruginosa and Candida albicans: do they really need to stick together?. Crit Care Med 2009; 37(3):1164-6.

20. Liu H. Co-regulation of pathogenesis with dimorphism and phenotypic switching in Candida albicans, a commensal and a pathogen. Int J Med Microbiol 2002; 292(5-6):299-311.

21. Saville SP, Lazzell AL, Monteagudo C, Lopez-Ribot JL. Engineered control of cell morphology in vivo reveals distinct roles for yeast and filamentous forms of Candida albicans during infection. Eukaryot Cell 2003; 2(5):1053-60

22. Balish E, Wagner RD. Probiotic bacteria for prophylaxis and therapy of candidiasis. Rev Iberoam Micol 1998; 15(4):261-4.
23. Payne S, Gibson G, Wynne A, Hudspith B, Brostoff J, Tuohy K. In vitro studies on colonization resistance of the human gut microbiota to Candida albicans and the effects of tetracycline and Lactobacillus plantarum LPK. Curr Issues Intest Microbiol 2003; 4(1):1-8.

24. Hogan DA, Kolter R. Pseudomonas-Candida interactions: an ecological role for virulence factors. Science 2002; 21;296(5576):2229-32.

25. Kerr J. Inhibition of fungal growth by Pseudomonas aeruginosa and Pseudomonas cepacia isolated from patients with cystic fibrosis. J Infect 1994; 28(3):305-10.

26. Foweraker J. Recent advances in the microbiology of respiratory tract infection in cystic fibrosis. Br Med Bull 2009; 89:93-110.

27. Tan MW, Rahme LG, Sternberg JA, Tompkins RG, Ausubel FM. Pseudomonas aeruginosa killing of Caenorhabditis elegans used to identify P. aeruginosa virulence factors. Proc Natl Acad Sci U S A 1999; 96(5):2408-13.

28. Tan MW, Ausubel FM. Caenorhabditis elegans: a model genetic host to study Pseudomonas aeruginosa pathogenesis. Curr Opin Microbiol 2000; 3(1):29-34.

29. Soll DR. Candida commensalism and virulence: the evolution of phenotypic plasticity. Acta Trop 2002; 81(2):101-10.

30. Brown DH Jr, Giusani AD, Chen X, Kumamoto CA. Filamentous growth of Candida albicans in response to physical environmental cues and its regulation by the unique CZF1 gene. Mol Microbiol 1999; 34(4):651-62.

31. Falleiros de Pádua RA, Norman Negri MF, Svidzinski AE, Nakamura CV, Svidzinski TI. Adherence of Pseudomonas aeruginosa and Candida albicans to urinary catheters. Rev Iberoam Micol 2008; 25(3):173-5.

32. Perepnikhatka V, Fischer FJ, Niimi M, Baker RA, Cannon RD, Wang YK, Sherman F, Rustchenko E. Specific chromosome alterations in fluconazole-resistant mutants of Candida albicans. J Bacteriol 1999; 181(13):4041-9.

33. Ruhnke M, Eigler A, Tennagen I, Geiseler B, Engelmann E, Trautmann M. Emergence of fluconazole-resistant strains of Candida albicans in patients with recurrent oropharyngeal candidosis and human immunodeficiency virus infection. J Clin Microbiol 1994; 32(9):2092-8.

34. White TC, Marr KA, Bowden RA. Clinical, cellular, and molecular factors that contribute to antifungal drug resistance. Clin Microbiol Rev 1998; 11(2):382-402.
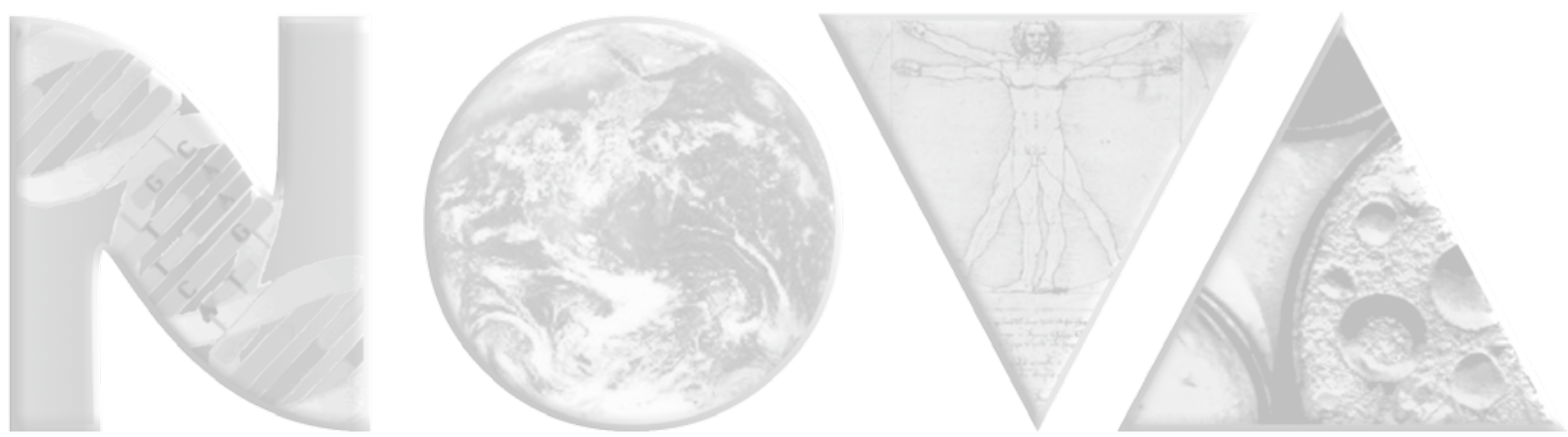